\title{
Parametric space for nonlinearly excited phase equation
}

\author{
D. V. Strunin ${ }^{1} \quad$ M. G. Mohammed ${ }^{2}$
}

(Received 5 January 2012; revised 6 June 2012)

\begin{abstract}
Slow variations in the phase of oscillators coupled by diffusion is generally described by a partial differential equation involving infinitely many terms. We consider the case of nonlocal coupling and numerically evaluate the ranges of parameters leading to different forms of a finite truncation of the equation, namely a form based on nonlinear excitation and a form based on linear excitation - the Kuramoto-Sivashinsky equation.
\end{abstract}

\section{Contents}

1 Introduction

C237

2 Complex nonlocal Ginzburg-Landau equation

C238

http://journal.austms.org.au/ojs/index.php/ANZIAMJ/article/view/5065 gives this article, (c) Austral. Mathematical Soc. 2012. Published June 18, 2012. ISSN 1446-8735. (Print two pages per sheet of paper.) Copies of this article must not be made otherwise available on the internet; instead link directly to this URL for this article. 
3 Numerical results

4 Conclusion

C245

References

$\mathrm{C} 247$

\section{Introduction}

In many situations reaction-diffusion systems can be reduced to systems of oscillators coupled by diffusion. At first sight, the diffusion should always equalise the phase of the oscillations throughout space. However, under certain conditions, the dynamics can be much more complex. Generally, an equation describing the dynamics of slow variations of the phase involves an infinite number of terms [1],

$$
\begin{aligned}
\partial_{\mathrm{t}} \psi= & \mathrm{a}_{1} \nabla^{2} \psi+\mathrm{a}_{2}(\nabla \psi)^{2} \\
& +\mathrm{b}_{1} \nabla^{4} \psi+\mathrm{b}_{2} \nabla^{3} \psi \nabla \psi+\mathrm{b}_{3}\left(\nabla^{2} \psi\right)^{2}+\mathrm{b}_{4} \nabla^{2} \psi(\nabla \psi)^{2}+\mathrm{b}_{5}(\nabla \psi)^{4} \\
& +\mathrm{g}_{1} \nabla^{6} \psi+\mathrm{g}_{2} \nabla^{5} \psi \nabla \psi+\mathrm{g}_{3} \nabla^{4} \psi \nabla^{2} \psi+\mathrm{g}_{4}\left(\nabla^{3} \psi\right)^{2}+\mathrm{g}_{5} \nabla^{4} \psi(\nabla \psi)^{2} \\
& +\mathrm{g}_{6}\left(\nabla^{2} \psi\right)^{3}+\mathrm{g}_{7} \nabla^{3} \psi \nabla^{2} \psi \nabla \psi+\mathrm{g}_{8} \nabla^{3} \psi(\nabla \psi)^{3}+\mathrm{g}_{9}\left(\nabla^{2} \psi\right)^{2}(\nabla \psi)^{2} \\
& +\mathrm{g}_{10} \nabla^{2} \psi(\nabla \psi)^{4}+\mathrm{g}_{11}(\nabla \psi)^{6} \\
& +\mathrm{e}_{1} \nabla^{8} \psi+\cdots
\end{aligned}
$$

Here $\psi$ is the phase, and $a_{\mathfrak{n}}, b_{\mathfrak{n}}, g_{\mathfrak{n}}, e_{\mathfrak{n}}, \ldots$ are constant coefficients. The righthand side of (1) is virtually a Taylor series in small parameter $\nabla^{2} \sim(1 / \mathrm{L})^{2}$, with $\mathrm{L}$ being the characteristic length presumed large. The balance of terms in (1) takes different forms depending on the magnitudes and signs of the coefficients. For example, when $a_{1}>0$ and the initial field of $\psi$ varies slowly in space, the diffusion term dominates during the entire period of evolution. In this case the equation is effectively reduced to the linear diffusion equation

$$
\partial_{t} \psi=a_{1} \nabla^{2} \psi, \text { where } a_{1}>0 .
$$


When $a_{1}=-\varepsilon<0$ is small and, by the order of magnitude, $a_{2}=1, b_{1}=-1$, Equation (1) reduces to the Kuramoto-Sivashinsky (KS) equation [2, 3],

$$
\partial_{\mathrm{t}} \psi=-\varepsilon \nabla^{2} \psi+(\nabla \psi)^{2}-\nabla^{4} \psi .
$$

The KS equation contains an anti-diffusion term $\left(-\varepsilon \nabla^{2} \psi\right)$, which represents self-excitation; it is counterbalanced by the dissipative term $\left(-\nabla^{4} \psi\right)$. Taking into account the smallness of $\varepsilon$, it is straightforward to show that the scales of $\psi$ and L resulting from the balance are such that the rest of the terms in (1) are negligible compared to the three balancing terms on the right-hand side of (3). Earlier $[4,5]$ we showed that a truncation based on nonlinear excitation is also possible,

$$
\partial_{\mathrm{t}} \psi=-\varepsilon \nabla^{2} \psi(\nabla \psi)^{2}+(\nabla \psi)^{4}+\nabla^{6} \psi .
$$

Equation (4), which we refer to as the nonlinearly excited phase (NEP) equation, is a reduced form of (1) when $b_{4}=-\varepsilon, b_{5}=1$ and $g_{1}=1$. The lower order terms preceding the above three on the right-hand side of (1) are negligible provided the coefficients $a_{1}, a_{2}, b_{1}, b_{2}$ and $b_{3}$ are small enough or zero. Thus, these five coefficients alongside with the coefficient $b_{4}$ must satisfy certain requirements of smallness [6]; therefore, we have six conditions in total.

We investigate the area in the space of independent parameters where (4) is indeed a valid truncation of (1). Also, out of curiosity, we compare this area with the area where a valid truncation is the KS equation.

\section{Complex nonlocal Ginzburg-Landau equation}

Consider a reaction-diffusion system [6] that captures qualitative features of some bio-systems, for example, cellular slime molds. Mathematically it is a 
slightly enhanced system compared to that of Tanaka and Kuramoto [7],

$$
\begin{aligned}
& \partial_{t} \mathbf{X}=\mathbf{f}(\mathbf{X})+\hat{\delta} \nabla^{2} \mathbf{X}+k_{1} g_{1}\left(S_{1}\right)+k_{2} g_{2}\left(S_{2}\right), \\
& \tau_{1} \partial_{t} S_{1}=-S_{1}+D \nabla^{2} S_{1}+h_{1}(\mathbf{X}) \\
& \tau_{2} \partial_{t} S_{2}=-S_{2}+D \nabla^{2} S_{2}+h_{2}(\mathbf{X})
\end{aligned}
$$

where $\hat{\delta}, k_{1}, k_{2}, \tau_{1}, \tau_{2}$ and $D$ are constants, and $\mathbf{X}, S_{1}$ and $S_{2}$ represent the concentrations of reactants. Equations (5)-(7) lead to a Ginzburg-Landau equation for the complex amplitude $A$ (which, loosely, is a measure of the concentrations) with two nonlocal terms,

$$
\begin{aligned}
\partial_{t} A= & \mu \sigma A-\beta|A|^{2} A+\delta \nabla^{2} A+k_{1} \eta_{1}^{\prime} \int d r^{\prime} G_{1}\left(r-r^{\prime}\right) A\left(r^{\prime}, t\right) \\
& +k_{2} \eta_{2}^{\prime} \int d \mathbf{r}^{\prime} G_{2}\left(r-r^{\prime}\right) A\left(r^{\prime}, t\right),
\end{aligned}
$$

where $G_{n}$ are coupling functions due to the presence of chemicals $S_{1}$ and $S_{2}$. In one dimension,

$$
\mathrm{G}_{\mathrm{n}}(x)=\frac{1}{2}\left(\zeta_{\mathrm{n}}+\mathrm{i} \eta_{\mathrm{n}}\right) e^{-\left(\zeta_{n}+i \eta_{n}\right)|x|}, \quad \mathrm{n}=1,2
$$

with

$$
\zeta_{n}=\left(\frac{1+\sqrt{1+\theta_{n}^{2}}}{2 D}\right)^{1 / 2} \text { and } \eta_{n}=\left(\frac{-1+\sqrt{1+\theta_{n}^{2}}}{2 D}\right)^{1 / 2} .
$$

All the new parameters in (8)-(10) are constants. Rescaling $(8)[6,7]$ leads to

$$
\begin{aligned}
\partial_{t} A= & A-\left(1+i c_{2}\right)|A|^{2} A+\left(\delta_{1}+i \delta_{2}\right) \nabla^{2} A \\
& +K_{1}\left(1+i c_{11}\right) \int d r^{\prime} G_{1}\left(r-r^{\prime}\right)\left[A\left(\mathbf{r}^{\prime}\right)-A(\mathbf{r})\right] \\
& +K_{2}\left(1+i c_{12}\right) \int d r^{\prime} G_{2}\left(r-r^{\prime}\right)\left[A\left(r^{\prime}\right)-A(r)\right] .
\end{aligned}
$$


Here, $\delta_{1}$ - the real part of $\delta$ - expresses the intensity of the diffusion, and $K_{1}$ and $K_{2}$ are rescaled $k_{1}$ and $k_{2}$ respectively. It is important for our analysis that Equation (11) contains the nine independent parameters: $\delta_{1}, \delta_{2}, c_{11}, c_{12}$, $\mathrm{c}_{2}, \mathrm{~K}_{1}, \mathrm{~K}_{2}, \theta_{1}$ and $\theta_{2}$.

The complex amplitude $A$ is connected to the real-valued amplitude, $\mathbf{a}$, and real-valued phase of the oscillations, $\varphi$, via $A=a e^{-i \varphi}$. Dynamically, the terms $A-\left(1+i c_{2}\right)|A|^{2} A$ in (11) make $a$ approach one, driven by $\partial_{t} a=a-a^{3}$. Simultaneously the phase increases at a constant rate as $\varphi=c_{2} t$. However, due to the rest of the terms in (11), the phase is not exactly equal to $c_{2} t$, so

$$
\varphi=c_{2} t+\psi .
$$

It can be shown that the phase departure, $\psi$, obeys Equation (1); the latter was derived [6] from the Ginzburg-Landau equation (11) by decomposing all the terms in a power series in $\nabla$. As a result, each coefficient in equation (1) emerges as a combination of the nine independent parameters. It was shown $[6$, 7] that the parameters $K_{1}$ and $K_{2}$ must satisfy the restriction

$$
\mathrm{K}_{1}+\mathrm{K}_{2}<1
$$

Another important restriction to meet is that the term $g_{1} \nabla^{6} \psi$ be dissipative, hence

$$
g_{1}>0
$$

\section{$3 \quad$ Numerical results}

Earlier we demonstrated [6] that there exist such values of the nine independent parameters, $\theta_{1}, \theta_{2}, c_{12}, c_{11}, c_{2}, K_{1}, K_{2} \delta_{1}$ and $\delta_{2}$, that the six conditions of smallness are satisfied, specifically, the smallness of $b_{4}=-\varepsilon$ and smallness of $a_{1}, a_{2}, b_{1}, b_{2}$ and $b_{3}$. Additionally, the restrictions $g_{1}>0, \delta_{1}>0$ and $K_{1}+K_{2}<1$ must be satisfied. To save space we do not present the cumbersome expressions of $a_{1}, a_{2}, b_{1}, b_{2}, b_{3}, b_{4}$ and $g_{1}$ in terms of the 
independent parameters but refer to earlier work [6] where these expressions are presented. We wrote a computer program in REDUCE language and computed the values of the parameters by solving the system of equations representing the smallness conditions. We also computed the values of the parameters that have to satisfy restrictions (13) and (14). Below we present just one set of computed values as an example.

The code works better when we present the small $b_{4}=-\varepsilon$ as a product of one of the unknowns, say $K_{1}$, and a small number, for example $b_{4}=-K_{1} \cdot 0.00001$. For the input values $\theta_{1}=5, \theta_{2}=2, c_{12}=1$ the computed output is: $\delta_{1}=27$, $\mathrm{K}_{1}=49, \mathrm{~K}_{2}=-89, \mathrm{~g}_{1}=33$. We are satisfied to see that all the restrictions are met,

$$
g_{1}>0, \quad \delta_{1}>0 \text { and } \quad K_{1}+K_{2}<1 .
$$

Clearly we can make $b_{4}$ as close to zero as we wish. Thus, for the above values of the independent parameters the NEP equation is a valid truncation of the phase equation (1).

Now we seek a range of the values of the parameters for which the NEP equation remains valid. Similarly to the numerical example, we execute the following procedure. We assign values to three of the independent parameters, choosing $\theta_{1}, \theta_{2}$ and $\mathbf{c}_{12}$. While these particular parameters have no a priori preference over the other, our numerical code appears to work (that is, guarantees convergence) only for certain chosen parameters. Thus, with the above parameters prescribed, we computed the values of the other six parameters from the list of nine. Finally, we inspect whether all the restrictions are met, and, based on the outcome, conclude whether or not a particular point in the $3 \mathrm{D}$ space $\left(\theta_{1}, \theta_{2}, \boldsymbol{c}_{12}\right)$ is one where the NEP equation is valid. If at least one of the restrictions is violated, the NEP equation is not valid. All the points fall into the three groups:

- the points where the NEP equation is valid, displayed as ' $\mathrm{O}$ ';

- the points where the equation is not valid, displayed as ' $*$ '; and

- the points, displayed as $\square$, for which we were unable to draw a conclusion 
about their validity as the computation took too long to finish.

For brevity we call the circles 'valid' points and the stars 'invalid'.

For the reaction-diffusion system in question, we also investigated the validity of the KS equation. The aim was to get some idea of how different the validity areas are for the KS and NEP equations, keeping in mind that the latter carries considerably stricter conditions. The KS equation is valid when only one condition on the coefficients is imposed,

$$
a_{1}=-\varepsilon<0,
$$

and, additionally, the following restrictions are satisfied,

$$
\mathrm{b}_{1}<0, \quad \delta_{1}>0 \text { and } \quad \mathrm{K}_{1}+\mathrm{K}_{2}<1 .
$$

Out of the nine independent parameters we had freedom to choose eight so that the remaining parameter was to be computed from Equation (16). We chose this computed parameter to be $\delta_{1}$. Again we consider the $3 \mathrm{D}$ space $\left(\theta_{1}, \theta_{2}, c_{12}\right)$. As we move from point to point along the line, the values of the three parameters change. For each point we assign values to the other five free parameters at our disposal, $\mathrm{c}_{11}, \mathrm{c}_{2}, \mathrm{~K}_{1}, \mathrm{~K}_{2}$ and $\delta_{2}$. We opted to take these values from the NEP computational experiments, that is, from the experiments executed at the same values of $\theta_{1}, \theta_{2}$ and $c_{12}$. Lastly we need to inspect if the restrictions (17) hold. If they do, the KS equation is valid at a given point, otherwise it is not.

Figures $1-5$ show the results along some randomly chosen lines (a more complete investigation is under way). We make the following observations. For the NEP equation in Figure 1, the lower and upper ends of the line consist of invalid points only - they extend to infinity. Only a short part of the shown segment is made of valid points. For the KS equation, the lower end is made of invalid points while the middle section has intermittent structure and the upper end consists of valid points. This implies that the distance occupied by the valid points is longer for the Ks line. Figure 2 shows a line oriented horizontally. The NEP line consists of valid points in its middle part with an 

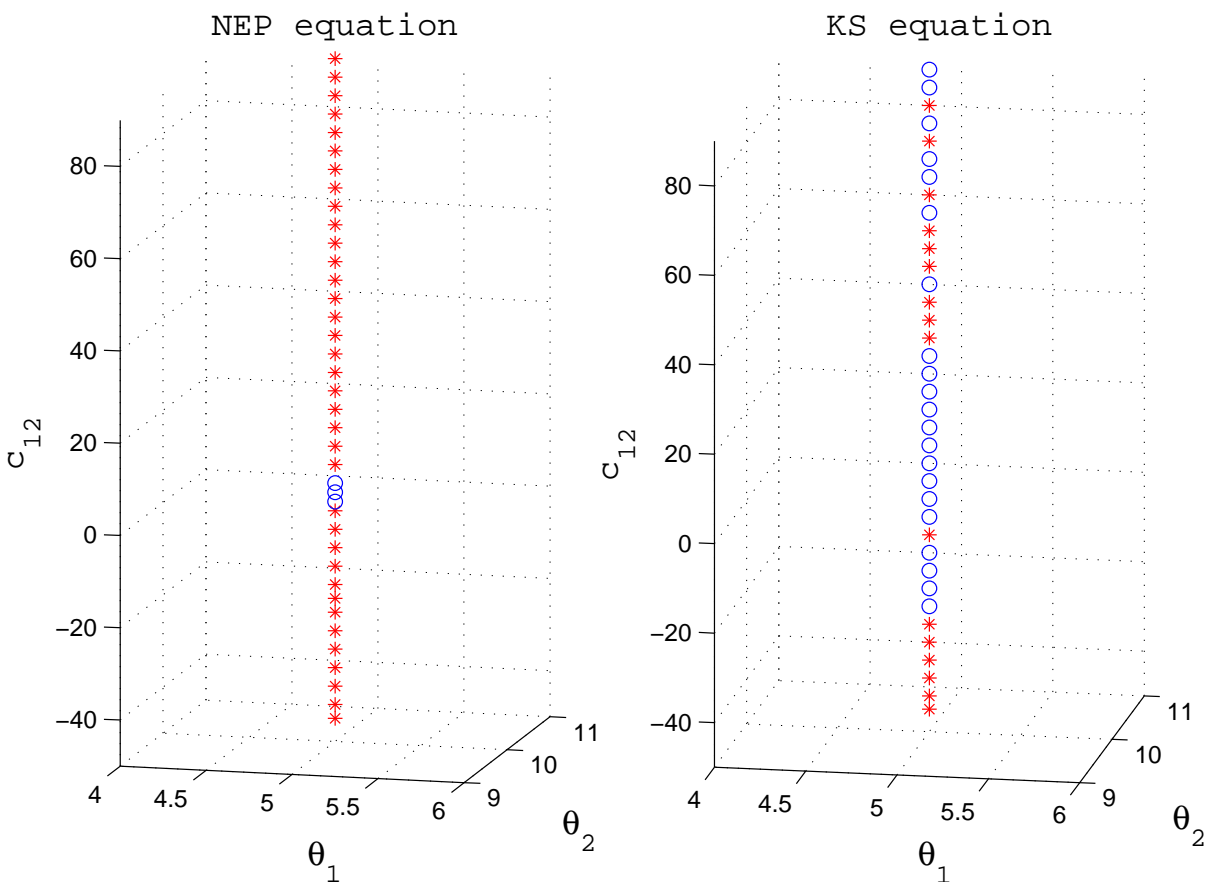

Figure 1: Valid and invalid points for the NEP and KS equations along a selected line.

inclusion of invalid points; along the ends it contains invalid points stretching to infinity. By contrast, the KS line has ends made of valid points only. This means that the distance over the entire (infinite) line covered by the valid points are longer for the KS equation than that for the NEP equation. Both Figure 1 and Figure 2 seem unsurprising considering the KS equation results from more relaxed conditions for the coefficients of the general phase equation. In Figure 3, for the both NEP and KS cases the line is made of a continuous set of invalid points at the lower end, while the upper end is made of valid points only; however, the KS line includes some invalid points in between. Therefore, for the shown stretch of the line, the distance occupied by the valid 
NEP equation

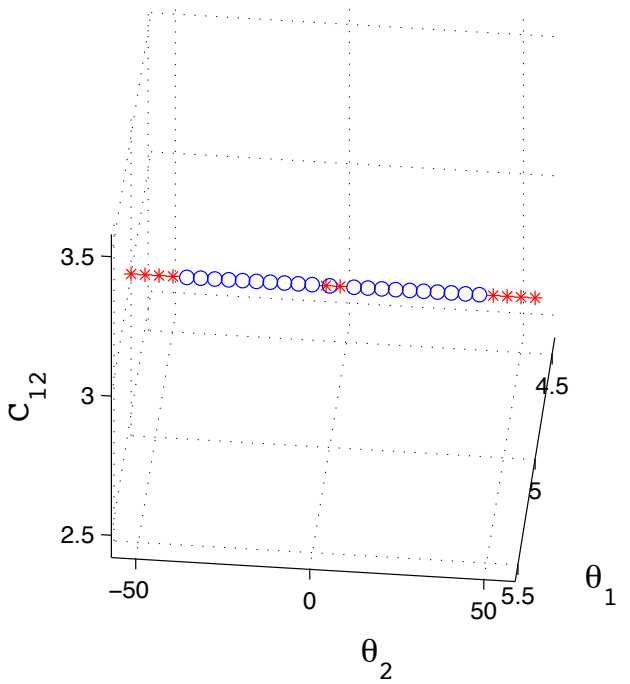

KS equation

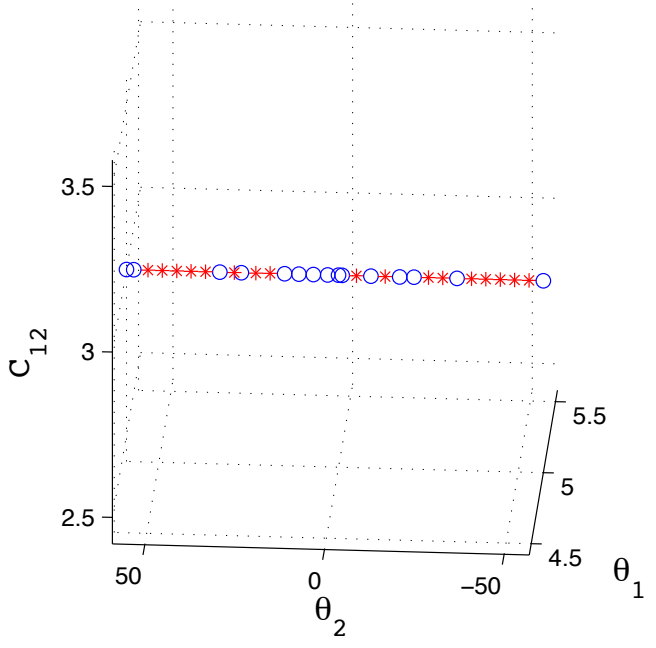

Figure 2: A comparison along a different line.

points is longer for the NEP equation than for the KS equation. In Figure 4 we again observe a common feature between the lines: their lower ends consist of invalid points. As in Figure 3, the KS line has inclusions of invalid points in the middle section. Above certain - approximately equal - heights on the lines we see uncertain points labelled by $\square$, for which we were unable to make a conclusion about validity. If we disregard the uncertain points, then the valid points for the NEP equation appear to occupy a longer segment of the shown piece of the line than they do for the KS equation. The structure of a horizontal line in Figure 5 for the NEP equation appears to be less intermittent than that for the KS equation; however, the continua of valid points for the both equations are comparable in length. 

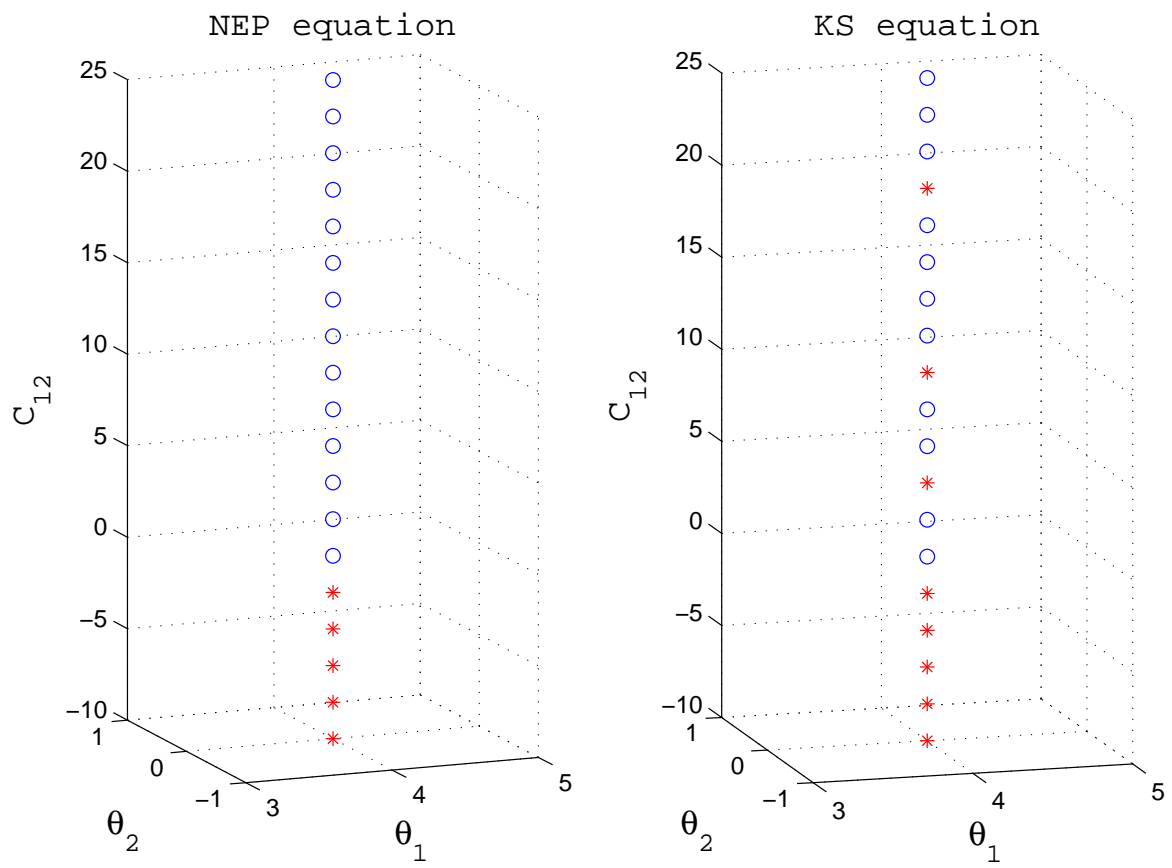

Figure 3: A comparison along a different line.

\section{Conclusion}

Exploring a 9D space of independent parameters for a system of nonlocally coupled oscillators is time consuming, so our analysis is by no means exhaustive. A possible approach for a more complete investigation can be based on a dense grid of lines piercing the 9D space. At this stage we determined only few intervals of validity of the nonlinearly excited phase equation and, for comparison, the KS equation. We pierced the $8 \mathrm{D}$ subspace spanned by the parameters $\theta_{1}, \theta_{2}, c_{12}, c_{11}, c_{2}, K_{1}, K_{2}$ and $\delta_{2}$ by a few lines. Of course we were only able to graphically display a $3 \mathrm{D}$ subsubspace, spanned by $\theta_{1}, \theta_{2}$ and $\mathbf{c}_{12}$. However, we emphasise that the lines in the $8 \mathrm{D}$ subspace were identical for 

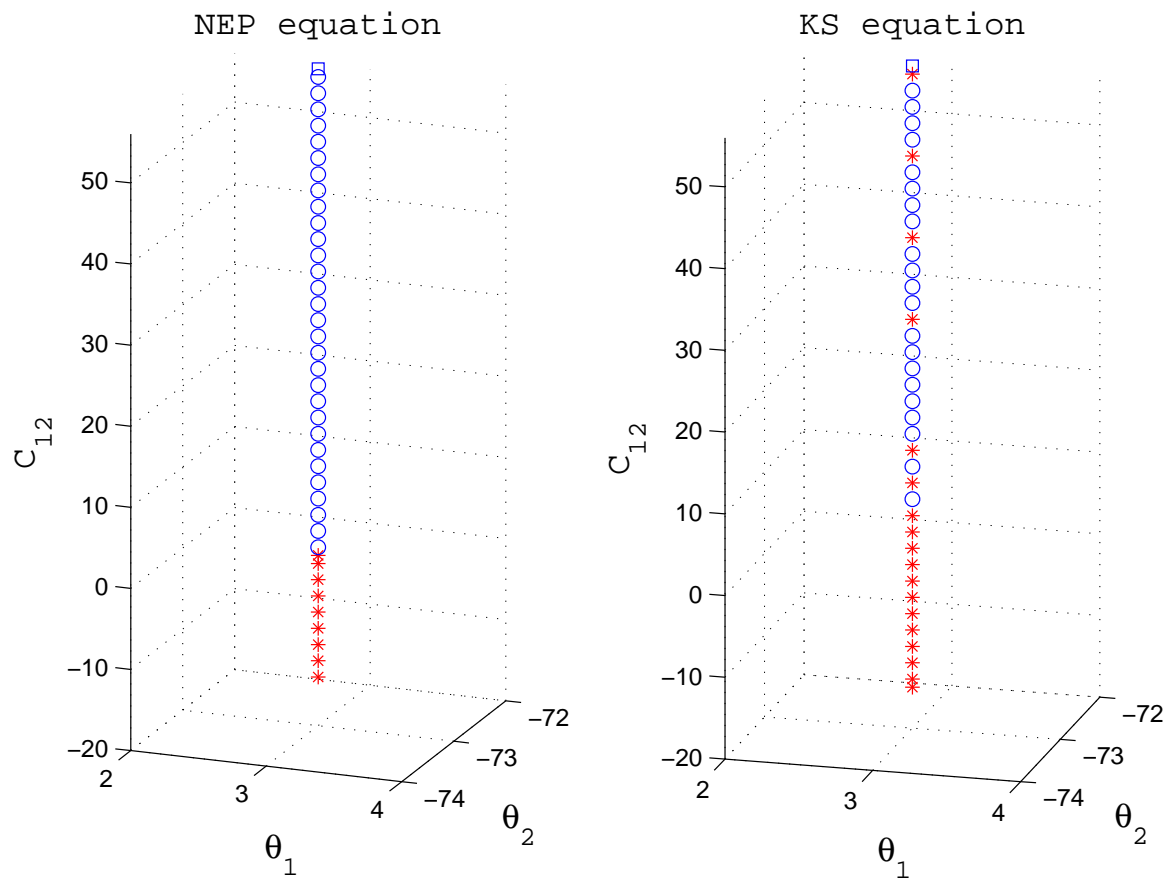

Figure 4: A comparison along a different line.

both equations. At each point on the line a given equation is either valid or invalid and sometimes our result was inconclusive due to computational limitations. Qualitatively, in spite of the many conditions on the coefficients of the general phase equation, the NEP equation has an appreciable range of validity in the 3D subsubspace. Recall that the reaction-diffusion systems considered in this article involves only two nonlocality inducing chemicals, $S_{1}$ and $S_{2}$. The validity range would expand if one adds more chemicals of this kind into the model and thereby bring more degrees of freedom. 

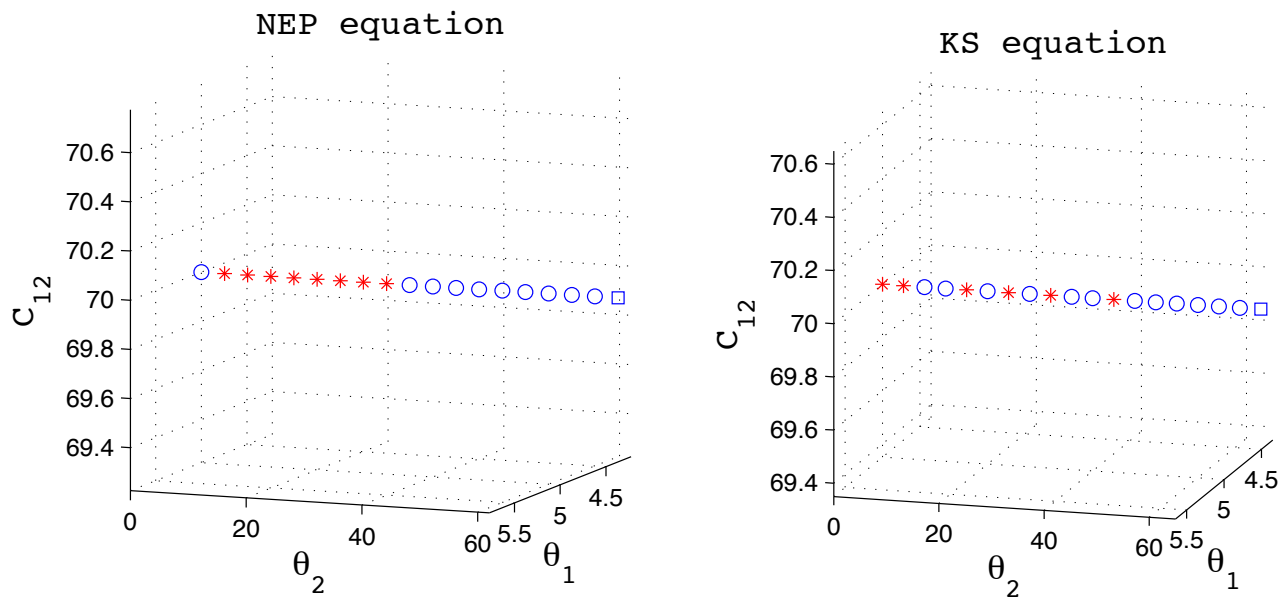

Figure 5: A comparison along a different line.

\section{References}

[1] Y. Kuramoto, Chemical Oscillations, Waves, and Turbulence. Springer-Verlag, Berlin, 1984. C237

[2] Kuramoto, Y. and Tsuzuki, T., Persistent propagation of concentration waves in dissipative media far from thermal equilibrium, Progr. Theor. Phys., 55, 1976, 356-369. doi:10.1143/PTP.55.356 C238

[3] Sivashinsky, G. I., Nonlinear analysis of hydrodynamical instability in laminar flames, Acta Astronaut., 4, 1977, 1177-1206. doi:10.1016/0094-5765(77)90096-0 C238

[4] Strunin, D. V., Autosoliton model of the spinning fronts of reaction, IMA J. Appl. Math., 63, 1999, 163-177. doi:10.1093/imamat/63.2.163 $\mathrm{C} 238$

[5] Strunin, D. V., Nonlinear instability in generalised nonlinear phase diffusion equation, Progr. Theor. Phys. Suppl., N 150, 2003, 444-448. 


\section{doi:10.1143/PTPS.150.444 C238}

[6] Strunin, D. V., Phase equation with nonlinear excitation for nonlocally coupled oscillators, Physica D: Nonlinear Phenomena, 238, 2009, 1909-1916. doi:10.1016/j.physd.2009.06.022 C238, C239, C240, C241

[7] Tanaka, D. and Kuramoto, Y., Complex Ginzburg-Landau equation with nonlocal coupling, Phys. Rev. E, 68, 2003, 026219. doi:10.1103/PhysRevE.68.026219 C239, C240

\section{Author addresses}

1. D. V. Strunin, Department of Mathematics and Computing, University of Southern Queensland 4350, Australia. mailto:strunin@usq.edu.au

2. M. G. Mohammed, Department of Mathematics and Computing, University of Southern Queensland 4350, Australia. mailto:MayadaGassabMohammed.Al-Badri@usq. edu .au 